\title{
Cerebral Metabolic Response to Neonatal Hypoglycemia in Growth-Retarded Dogs
}

\author{
ROBERT M. KLIEGMAN \\ Department of Pediatrics, Case Western Reserve University School of Medicine, Rainbow Babies and Childrens \\ Hospital, Cleveland, Ohio 44106
}

\begin{abstract}
The cerebral metabolic effects of hypoglycemia due to intrauterine growth retardation were studied in newborn dogs. Intrauterine growth retardation was induced in newborn dogs after 3 days of maternal nutritional deprivation (birth weight $251 \pm 7$ versus $227 \pm 7$ g, $p<$ $0.01)$. After birth, growth retarded pups developed fasting neonatal hypoglycemia which lasted from 3 to $9 \mathrm{~h}$ of life. The cerebral arteriovenous differences for glucose, oxygen, and ketone bodies were not different between growthretarded pups or those from age-matched controls. The cerebral venous efflux of lactate was reduced, whereas the extraction of glucose (relative to blood glucose) was enhanced among growth-retarded pups. Cerebral glycogen content was lower in pups with growth retardation whereas phosphoenolpyruvate and pyruvate concentrations were augmented among growth-retarded pups. The latter may reflect a more oxidized cytoplasmic redox state but may also be due to diminished lactate efflux from the brain. Cerebral ATP content was not affected during periods of reduced blood glucose levels. These results suggest that in newborn dogs hypoglycemia associated with intrauterine growth retardation alters cerebral metabolism by increasing cerebral extraction of glucose and decreasing CNS efflux of lactate. We speculate that the net effect is increased lactate utilization within oxidative pathways and preservation of cerebral oxygen uptake. Cerebral glucose utilization is directed away from glycogen synthesis and toward glycolysis. Lactate oxidation rather than release to the systemic circulation may maintain cerebral ATP production in growth-retarded hypoglycemic newborn dogs. (Pediatr Res 24: 649-652, 1988)
\end{abstract}

\section{Abbreviations}

MCS, maternal canine starvation BHBA, $\beta$-hydroxybutyrate

Fasting neonatal hypoglycemia is a common manifestation of altered fuel metabolism among mammals with intrauterine growth retardation (1-3). Maternal nutritional deprivation results in fetal growth retardation and fasting hypoglycemia among newborn dogs $(1,2)$. Hypoglycemia may have a significant affect on the developing CNS as severe neurologic sequalae have been reported after symptomatic neontal hypoglycemia (3). Nonetheless, asymptomatic hypoglycemia is associated with no or minimal sequalae, thus suggesting the possibility of metabolic adaptation to low circulating glucose levels (3-5). Indeed in adult rats

Received March 16, 1988; accepted July 25, 1988.

Correspondence Robert M. Kliegman, M.D., Rainbow Babies and Childrens Hospital, 2101 Adelbert Road, Cleveland, OH 44106.

Supported by NIH Grant HD20851. rendered hypoglycemic by injection of insulin, the cerebral oxygen consumption remains unchanged despite lower blood glucose levels (4-7). Oxidation of alternate fuels or enhanced extraction of glucose from the circulation may provide substrate to permit unperturbed rates of cerebral oxygen consumption during hypoglycemia (7).

A previous report from our laboratory had demonstrated that fasting hypoglycemia in growth-retarded newborn dogs resulted in lower cerebral glycogen and glucose content but did not alter cerebral ATP levels (8). The adaptive metabolic responses were not identified and the contribution of alternate fuels to cerebral oxidative metabolism was not assessed. In the present study of growth-retarded newborn dogs we evaluated the cerebral arteriovenous differences for cerebral fuels and oxygen simultaneously with cortical metabolites to determine the effects of hypoglycemia on cerebral glucose utilization.

\section{MATERIALS AND METHODS}

All enzymes, reagents, and standards were purchased from Sigma Chemical Co., St. Louis, MO.

Experimental design and animal care. Pregnant beagle dogs were maintained in kennels and fed a standard canine diet. Before delivery, seven mothers were fasted overnight $(18 \mathrm{~h}$ ) providing the control group, whereas nine were fasted for 3 days providing the MCS group $(1,8)$. Cesarean section was performed at term ( $60 \pm 2$ days). Immediately before the cesarean section, intravenous anesthesia $(5 \mathrm{mg} / \mathrm{kg}$ chlorpromazine) was given to the mother. Lidocaine ( $2 \%$ ) was used to anesthetize the incision area. The newborn pups were immediately transferred to an incubator maintained at $37^{\circ} \mathrm{C}$ with relative humidity of $70 \%$. The newborn pups were permitted to stabilize, were weighed, and had umbilical arterial and venous catheters (3.5 French Argyle, St. Louis, MO) placed atraumatically. This was to facilitate blood withdrawal and quantitation of turnover data as reported in a separate report. The newborn pups were fasted throughout the study period in both groups. This protocol was approved by the Case Western Reserve University Animal Welfare Committee.

Before tissue sampling blood was withdrawn from the aorta and simultaneously from the superior sagittal sinus after percutaneous cannulaton via the anterior fontanel. Thereafter the pups from each litter were killed. The entire cerebral cortex was freezeclamped at the temperature of liquid nitrogen in less than $3 \mathrm{~s}$ with heavy aluminum blocks. These blocks were previously cooled to the temperature of liquid nitrogen. In addition, the blocks were placed at the end of bone clamps to ensure immediate flattening of the cortex. This avoids differential cooling of the center of the sample compared with the external surface. Thickness of frozen samples was between 1 and $2 \mathrm{~mm}$. Redox state and ATP levels in the normal pup group do not demonstrate any evidence for brain ischemia $(9,10)$. The tissues were stored at $-80^{\circ} \mathrm{C}$ until analyzed. 
Analyses. The frozen cerebral cortex was pulverized to a fine powder at the temperature of dry ice. Extraction and homogenization were performed as reported before at $0^{\circ} \mathrm{C}(2,8)$. Immediately after acid extraction and rapid neutralization, aliquots were divided and stored at $-80^{\circ} \mathrm{C}$. ATP was analyzed within $1 \mathrm{wk}$ of storage of the frozen tissue and within $1 \mathrm{wk}$ of extraction and neutralization. These periods of storage have not resulted in a decrement of ATP levels among neonatal canine brain samples. Our results for ATP in normal neonatal dogs are not different from those values reported by others in newborn dogs and rodents $(9,10)$. Thereafter, glycogen, glucose, UDP glucose, glucose-6-phosphate, fructose-6-phosphate, phosphoenolpyruvate, pyruvate, and lactate were assayed as reported previously $(2,8)$. Cerebral cytoplasmic NAD/NADH ratio was calculated as described before $(2,8)$. All analyses were performed in duplicate. The interassay variation was $5-10 \%$ whereas the intraassay was less than $5 \%$. Oxygen contents was determined

Table 1. Circulating fuels $(m M)$ (mean $\pm S E)$

\begin{tabular}{lcccc}
\hline & \multicolumn{4}{c}{ Postnatal age (h) } \\
\cline { 2 - 5 } & 3 & 6 & 9 & 24 \\
\hline Glucose & & & & \\
Control & $3.48 \pm 0.34$ & $4.89 \pm 0.59$ & $6.05 \pm 0.78$ & $4.50 \pm 0.49$ \\
$n$ & 10 & 11 & 12 & 9 \\
MCS & $2.24 \pm 0.25^{*}$ & $2.19 \pm 0.34 \dagger$ & $3.59 \pm 0.43 \dagger$ & $3.42 \pm 0.60$ \\
$n$ & 13 & 14 & 14 & 9 \\
& & & & \\
Lactate & & & & \\
Control & $2.30 \pm 0.44$ & $1.63 \pm 0.20$ & $1.64 \pm 0.23$ & $1.92 \pm 0.26$ \\
MCS & $1.98 \pm 0.30$ & $1.51 \pm 0.16$ & $1.64 \pm 0.13$ & $1.61 \pm 0.28$ \\
\hline
\end{tabular}

$* p<0.02$.

$\dagger p<0.01$. on the Radiometer hemoximeter (Radiometer America Inc., Westlake, OH).

Statistical analyses. Tabular notations are the mean \pm SEM. Sample size is depicted in Table 1-4. Tissue metabolites are expressed as $\mu \mathrm{mol} / \mathrm{g}$ tissue wet weight. Circulating fuels are expressed as mM. Standard calculator programs were used for Student's $t$ test analyses of data between the two groups. Due to normal changes with age in the 1st day of life, the major comparisons are made between experimental and control groups at the same time period.

\section{RESULTS}

Circulating fuels. Three days of MCS reduced birth weight by $10 \%(251 \pm 7$ versus $277 \pm 7 \mathrm{~g}, p<0.01)$. After birth, growthretarded pups demonstrated significantly lower blood glucose levels at 3,6 , and $9 \mathrm{~h}$ of fasting (Table 1). Blood lactate levels were not different between the groups at any time interval during the periods of neonatal fasting.

Cerebral arteriovenous differences. Despite significantly lower blood glucose levels the cerebral arteriovenous differences for glucose were similar at each time point studied among both pup groups (Table 2). Maintenance of the cerebral arteriovenous difference for glucose in the presence of significantly lower circulating glucose levels was dependent on a higher rate of cerebral glucose extraction as noted at 3,6 , and $9 \mathrm{~h}$ of age in pups after MCS (Table 2).

The cerebral uptake of alternate fuels (acetoacetate and BHBA) were similar at each age-matched time point among both groups of pups (Table 2). Nonetheless the efflux of lactate from the sagittal sinus was significantly reduced among growth-retarded pup at 3,6 , and $9 \mathrm{~h}$ of age compared with appropriately agematched control pups (Table 2). At each of these time points it appeared that more lactate was retained within the CNS in growth-retarded pups than among control dogs.

Table 2. Cerebral arteriovenous differences after $M C S^{*}$

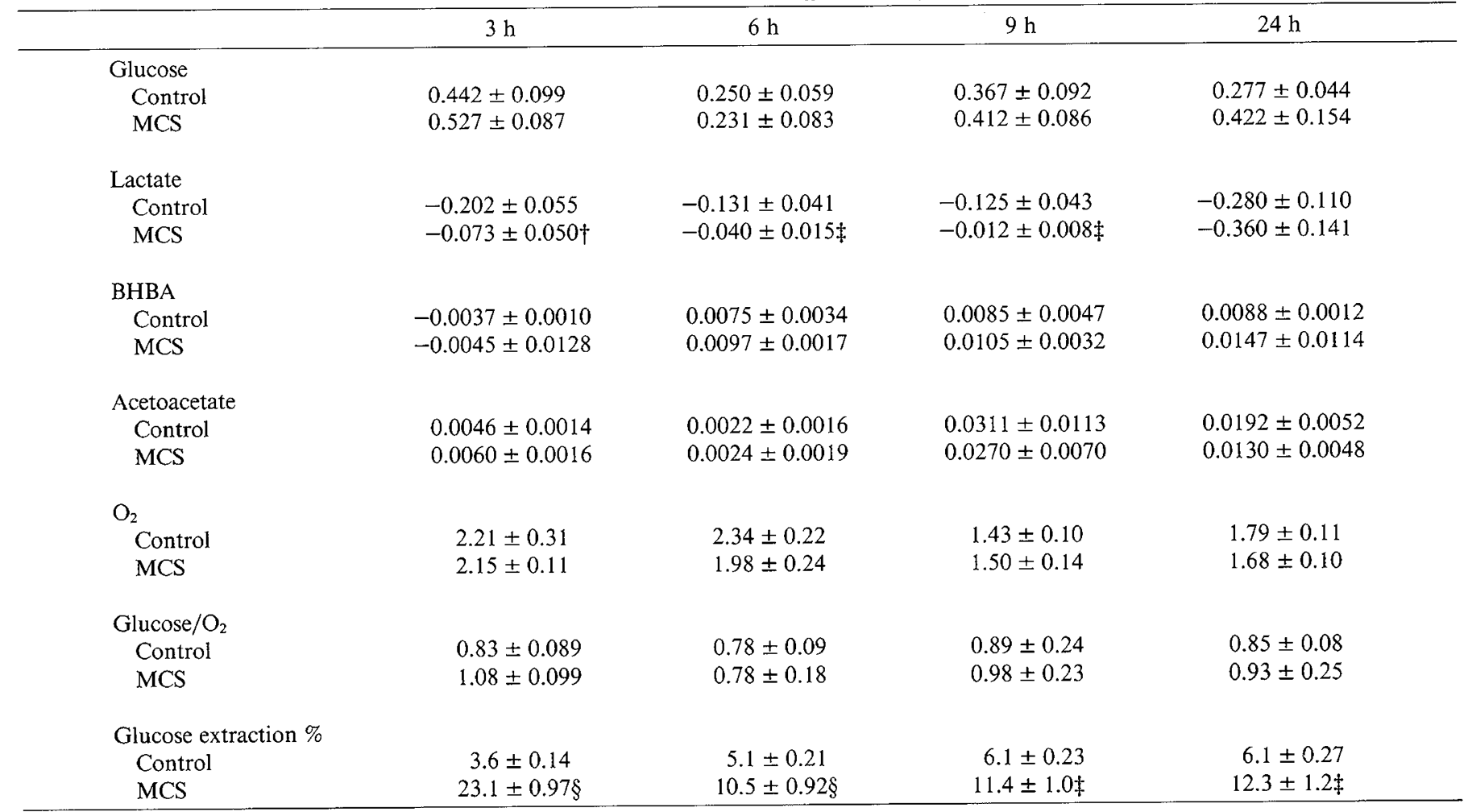

$*$ Glucose $/ \mathrm{O}_{2}=6$ xart-venous glucose $(\mathrm{mM}) /$ art-venous $\mathrm{O}_{2}(\mathrm{mM}) ; n$ as in Table 1.

$\dagger p=0.02$.

$\ddagger p=0.01$.

$\S p=0.001$. 
Cerebral oxidative metabolism as assessed by the arteriovenous difference for oxygen and the glucose to oxygen quotient was not affected by MCS and fasting neonatal hypoglycemia (Table 2). Nonetheless if the cerebral glucose:oxygen quotient is corrected by the carbon efflux of lactate it would appear that more of the oxygen used by the brain of the growth-retarded pup was associated with glucose oxidation (assuming the lactate efflux is derived from partial metabolism of glucose).

Cerebral intermediates. Cerebral cortical levels of glucose were reduced throughout the study period in growth-retarded pups (Table 3). At 3,6, and $9 \mathrm{~h}$ of age this reflected the low glucose values in the circulation. Cerebral glycogen content was reduced in growth retarded pups at 3,6 , and $24 \mathrm{~h}$ of age and was associated with an elevation of UDP-glucose during the entire study period (Table 3). Although there were no changes of cerebral glucose 6phosphate, fructose 6-phosphate, and lactate levels, phosphoenolpyruvate and pyruvate concentrations were higher at each time point studied in growth-retarded pups (Table 3). This may reflect in part the more oxidized cytoplasmic redox state noted in growth-retarded pups between 3 and $9 \mathrm{~h}$ of age (Table 4). During the entire study period there were no perturbations of the cerebral ATP concentration (Table 4).

\section{DISCUSSION}

In the present study fasting neonatal hypoglycemia in intrauterine growth-retarded dogs was associated with no alterations of cerebral oxygen or glucose arteriovenous differences or tissue ATP concentration. Despite reduced circulating glucose levels the arteriovenous difference of glucose was maintained compa- rable to that in control pups because the cerebral extraction of glucose was augmented in growth-retarded hypoglycemia pups. The ability to increase the cerebral extraction of glucose appears to be an important mechanism to maintain the availability of glucose to the developing CNS (11-13).

The observation of similar ratios of cerebral glucose and oxygen arteriovenous differences between pup groups suggests that the availability of these substances were not rate limiting. Although not determined in the present study, cerebral blood flow alterations must also be taken into consideration, in making the assumption that the arteriovenous differences qualitatively reflect actual cerebral uptake and utilization. In previous investigations in our laboratory on the effects of hypoglycemia on cerebral blood flow and cerebral glucose utilization we were unable to demonstrate an effect of hypoglycemia on cerebral

Table 4. Cerebral energy state*

\begin{tabular}{lcccc}
\hline & $3 \mathrm{~h}$ & $6 \mathrm{~h}$ & $9 \mathrm{~h}$ & $24 \mathrm{~h}$ \\
\hline ATP $(\mu \mathrm{mol} / \mathrm{g})$ & & & & \\
Control & $2.39 \pm 0.11$ & $2.49 \pm 0.17$ & $2.36 \pm 0.25$ & $2.53 \pm 0.20$ \\
MCS & $2.39 \pm 0.20$ & $2.67 \pm 0.13$ & $2.38 \pm 0.11$ & $2.57 \pm 0.13$
\end{tabular}

$\mathrm{NAD} / \mathrm{NADH}$

$\begin{array}{lllll}\quad \text { cytoplasm } & & & & \\ \text { Control } & 1199 \pm 92 & 1045 \pm 45 & 1118 \pm 65 & 1107 \pm 50 \\ \text { MCS } & 1482 \pm 102 \dagger 1279 \pm 100 \dagger 1363 \pm 90 \dagger & 1104 \pm 95\end{array}$

$* n$ as in Table 1 .

$\dagger p=0.01$.

Table 3. Cerebral glycolytic intermediates ( $\mu \mathrm{mol} / \mathrm{g}$ tissue wet $w t$; mean $\pm S E M)^{*}$

\begin{tabular}{|c|c|c|c|c|}
\hline & $3 \mathrm{~h}$ & $6 \mathrm{~h}$ & $9 \mathrm{~h}$ & $24 \mathrm{~h}$ \\
\hline \multicolumn{5}{|l|}{ Glucose } \\
\hline Control & $2.72 \pm 0.34$ & $2.59 \pm 0.32$ & $3.21 \pm 0.46$ & $3.22 \pm 0.45$ \\
\hline MCS & $1.84 \pm 0.31 \dagger$ & $1.34 \pm 0.18 \dagger$ & $1.54 \pm 0.25 \dagger$ & $1.75 \pm 0.39 \dagger$ \\
\hline \multicolumn{5}{|l|}{ Glycogen } \\
\hline Control & $2.27 \pm 0.21$ & $2.10 \pm 0.16$ & $2.49 \pm 0.15$ & $2.34 \pm 0.14$ \\
\hline MCS & $2.01 \pm 0.18$ & $1.65 \pm 0.16+$ & $1.53 \pm 0.24 \uparrow$ & $1.85 \pm 0.11 \ddagger$ \\
\hline \multicolumn{5}{|c|}{ UDP glucose } \\
\hline Control & $0.067 \pm 0.007$ & $0.078 \pm 0.004$ & $0.078 \pm 0.003$ & $0.074 \pm 0.006$ \\
\hline MCS & $0.091 \pm 0.011 \ddagger$ & $0.089 \pm 0.003 \S$ & $0.098 \pm 0.012 \S$ & $0.093 \pm 0.006 \dagger$ \\
\hline \multicolumn{5}{|c|}{ Glucose-6-phosphate } \\
\hline Control & $0.099 \pm 0.006$ & $0.092 \pm 0.005$ & $0.104 \pm 0.008$ & $0.097 \pm 0.004$ \\
\hline MCS & $0.088 \pm 0.010$ & $0.087 \pm 0.009$ & $0.096 \pm 0.011$ & $0.091 \pm 0.006$ \\
\hline \multicolumn{5}{|c|}{ Fructose-6-phosphate } \\
\hline Control & $0.041 \pm 0.004$ & $0.044 \pm 0.007$ & $0.049 \pm 0.009$ & $0.042 \pm 0.008$ \\
\hline MCS & $0.048 \pm 0.009$ & $0.051 \pm 0.010$ & $0.048 \pm 0.008$ & $0.048 \pm 0.009$ \\
\hline \multicolumn{5}{|c|}{ Phosphoenolpyruvate } \\
\hline Control & $0.096 \pm 0.004$ & $0.089 \pm 0.005$ & $0.093 \pm 0.009$ & $0.098 \pm 0.008$ \\
\hline MCS & $0.128 \pm 0.006 \|$ & $0.118 \pm 0.007 \|$ & $0.119 \pm 0.005 \dagger$ & $0.115 \pm 0.004 \S$ \\
\hline \multicolumn{5}{|l|}{ Pyruvate } \\
\hline Control & $0.180 \pm 0.011$ & $0.178 \pm 0.014$ & $0.174 \pm 0.013$ & $0.161 \pm 0.011$ \\
\hline MCS & $0.254 \pm 0.015 \dagger$ & $0.223 \pm 0.013 \ddagger$ & $0.264 \pm 0.016 \dagger$ & $0.225 \pm 0.024 \ddagger$ \\
\hline \multicolumn{5}{|l|}{ Lactate } \\
\hline Control & $1.37 \pm 0.12$ & $1.61 \pm 0.12$ & $1.44 \pm 0.18$ & $1.38 \pm 0.13$ \\
\hline MCS & $1.75 \pm 0.25$ & $1.49 \pm 0.07$ & $1.89 \pm 0.26$ & $1.84 \pm 0.32$ \\
\hline
\end{tabular}

\footnotetext{
$* n$ as is Table 1 .

$\dagger p=0.01$.

$\ddagger p=0.02$.

$\S p=0.05$.

$\| p=0.001$
} 
blood flow, cerebral oxygen uptake, or cerebral glucose utilization (11). Similar observations have been noted in adult rats as reviewed by Siesjo (7). Given our present data we speculate that cerebral glucose and oxygen consumption were similar in the pup groups because the arteriovenous difference for glucose and oxygen were unperturbed.

Cerebral metabolite perturbations were noted and suggested that glucose utilization by the cerebral glycogen synthetic pathway was inhibited in growth-retarded pups. This was demonstrated by reduced tissue glycogen content and elevated UDPglucose concentrations in growth-retarded pups. The latter observation would be expected if glycogen synthase was less active in these pups resulting in a build-up of this metabolite proximal to the glycogen synthase step (7).

Cerebral glucose utilization in growth-retarded pups was more likely to occur by the glycolytic pathway as noted by increased PEP and pyruvate levels $(6,14-17)$. An additional explanation for these observations may relate to the reduced efflux of lactate and the more oxidized cerebral cytoplasmic redox state in growth-retarded pups. Less cerebral lactate efflux may reflect more complete cerebral oxidation of glucose rather than release of lactate derived from glucose before entering the Krebs cycle (14). Lactate has been demonstrated to be an oxidizable fuel for brain metabolism under different metabolic conditions (18). It is likely that one adaptive mechanism in these growth-retarded hypoglycemic pups is to release less lactate to the venous circulation and oxidize more lactate (hence glucose) in the brain. The redox state alterations may reflect oxidative ATP production and hence production of NAD from NADH during this process (16, 17). This would maintain cerebral ATP concentrations and result in the equivalent rates of cerebral oxygen consumption noted during hypoglycemia in this study and other investigations of hypoglycemia $(5,7)$.

The response of the growth-retarded newborn to hypoglycemia involves more than one adaptive mechanism, with the net result being a more efficient extraction and then intracerebral utilization of glucose for oxidation. First increased extraction of circulating glucose may result in glucose entry into the cortex. Next glucose may be diverted away from energy using pathways (glycogen synthesis) to energy-producing pathways (glycolysis) (17, 18). Finally lactate, a product of glycolysis, is further metabolized by the Krebs cycle rather than being released into the venous outflow of the CNS $(14,16)$. In the presence of similar rates of oxygen uptake, these metabolic-perturbations assure sufficient glucose to serve as the substrate for cerebral oxidative metabolism and ATP production.

In the present study hypoglycemia was of moderate severity and was not associated with clinical signs of brain dysfunction. The current data cannot be extrapolated to states of symptomatic hypoglycemia. Other models that demonstrated profound hypoglycemia with coma were associated with reduced cerebral ATP $(4,5,7,14)$. These previous studies suggested that a point may be reached when the proposed adaptive responses to hypoglycemia can no longer provide sufficient substrate to maintain cerebral energy production, and thus produce altered brain function.

\section{REFERENCES}

1. Kliegman R, Miettinen E, Adam P 1981 Fetal and neonatal responses to maternal canine starvation: circulating fuels and neonatal glucose production. Pediatr Res 15:945-951

2. Miettinen E, Kliegman R 1983 Fetal and neonatal responses to extended maternal canine starvation. II. Fetal and neonatal liver metabolism. Pediatr Res 17:639-644

3. Koivisto M, Blanco-Sequeiros M, Krause U 1972 Neonatal symptomatic and asymptomatic hypoglycaemia: a follow-up study of 151 children. Dev Med Child Neurol 14:603-614

4. Lewis L, Ljunggren B, Ratcheson R, Siesjo B 1974 Cerebral energy state in insulin induced hypoglycemia related to blood glucose levels. J Neurochem 23:673-679

5. Norberg K, Siesjo B 1976 Oxidative metabolism of cerebral cortex of the rat in severe insulin induced hypoglycemia. J Neurochem 26:345-352

6. Tews $\mathbf{J}$, Carter $\mathbf{S}$, Stone W 1965 Chemical changes in the brain during insulin hypoglycemia and recovery. J Neurochem 12:679-693

7. Siesjo BK 1978 Brain Energy Metabolism. John Wiley \& Sons, New York

8. Kliegman R, Miettinen E, Rolin W, Adam P 1981 Fetal and neonatal cerebra metabolism following maternal canine starvation. Pediatr Res 15:859-865

9. Vannucci R, Duffy T 1970 Influence of birth on carbohydrate and energy metabolism in rat brain. Am J Physiol 226:933-940

10. Vannucci R, Nardis E, Vannucci S 1980 Cerebral metabolism during hypoglycemia and asphyxia in newborn dogs. Biol Neonate 38:276-286

11. Huang M, Kliegman R, Voelker K, Kall D, Chau K 1987 Relationship of systemic glucose production to cerebral glucose utilization in newborn dogs. Pediatr Res 21:215A

12. Levitsky L, Fisher D, Paton J, Delannoy C 1977 Fasting plasma levels of glucose, acetoacetate, $d$ - $\beta$-hydroxybutyrate, glycerol, and lactate in the baboon infant: correlation with cerebral uptake of substrates and oxygen. Pediatr Res 11:298-302

13. Gregoire N, Gjedde A, Plum F, Duffy T 1978 Cerebral blood flow and cerebral metabolic rates for oxygen, glucose and ketone bodies in newborn dogs. $\mathrm{J}$ Neurochem 30:63-69

14. Agardh C, Folbergrova J, Siesjo B 1978 Cerebral metabolic changes in profound insulin induced hypoglycemia and the recovery period following glucose administration. J Neurochem 31:1135-1142

15. Llorente P, Marco R, Sols A 1970 Regulation of liver pyruvate kinase and the phosphoenolpyruvate crossroads. Eur J Biochem 13:45

16. Greenbaum AL, Fumaa KA, McLean P 1971 The distribution of hepatic metabolites and the control of the pathways of carbohydrate metabolism in animals of different dietary and hormonal status. Arch Biochem Biophys 143:617

17. Gumaa KA and McLean P 1969 The pentose phosphate pathway of glucose metabolism: enzyme profiles and transient and steady-state content of intermediates of alternative pathways of glucose metabolism in Krebs ascites cells. Biochem J 115:1009

18. Fernandez J, Berger R, Smit G 1984 Lactate as a cerebral metabolic fuel for glucose-6-phosphatase deficient children. Pediatr Res 18:335-339 